\title{
Adding explicit content classification to nonlinear filters
}

\author{
H. Hu • G. de Haan
}

Received: 20 June 2010 / Revised: 8 November 2010 / Accepted: 9 November 2010 / Published online: 11 January 2011

(C) The Author(s) 2011. This article is published with open access at Springerlink.com

\begin{abstract}
Nonlinear filters are known for better edgepreserving performance in image processing applications as they can adapt to some local image content. Instead of trying to find a single optimal filter that can adapt to all the image content, some classification-based approaches first apply a pre-classification on the image content and then employ an optimal linear filter for each content class. It is interesting to extend the linear filter in such approaches to a nonlinear filter and see if the explicit content classification, can still add to such inherently adapting nonlinear filters. In this paper, we investigate several categories of nonlinear filters: order statistics filters, hybrid filters, neural filters, and bilateral filters with different forms of content classification in various image processing applications, including image de-blocking, noise reduction, and image interpolation.
\end{abstract}

Keywords Nonlinear filter - Image processing $\cdot$ Content classification

\author{
H. $\mathrm{Hu}(\varangle)$ \\ Distributed Sensor System Group, Philips Research Laborotaries, \\ High Tech Campus 34, 5656 AE Eindhoven, The Netherlands \\ e-mail: hao.hu@philips.com \\ G. de Haan \\ Video and Image Processing Group, Philips Research Laborotaries, \\ High Tech Campus 36, 5656 AE Eindhoven, The Netherlands \\ e-mail: g.de.haan@philips.com \\ G. de Haan \\ Electrical Engineering Department, \\ Eindhoven University of Technology, \\ Den Dolech 2, 5600 MB Eindhoven, The Netherlands
}

\section{Introduction}

In image processing applications, linear filters are commonly used. They estimate the output by using the weighted sum of the pixel values in a local window. They have good performance at eliminating Gaussian noise, but they can blur image edges and details [1]. In order to solve the problem with linear filters, many nonlinear filters have been introduced. Order statistics filters $[2,3]$ perform better in smoothing tasks where edge preservation is important. The bilateral filter [4] has the ability to locally adapt the filtering to the image content. Regarded as a universal function approximator, the neural network can be used as a neural filter [5] which also inherently adapts to the local image content and combines linear and nonlinear ingredients.

As an alternative to solve the problem that a single linear filter is not optimal for different image content, some content adaptive filtering methods have been introduced, such as Kondo's method [6] and Atkins' method [7]. Kondo's method applies a pre-classification to classify the local image structure in the filter's aperture and uses an optimal linear filter for each class.

Therefore, it is interesting to introduce the content classification to the nonlinear filters and see if the explicit content classification can still add to these nonlinear filters, especially inherently adapting filters such as the bilateral filter. It is, therefore, interesting to answer the question whether the neural filter can profit from additional content classification and whether it is the ultimate trained nonlinear filter, or not.

To answer the above questions in this paper, we will study four different categories of nonlinear filters: order statistics filters, hybrid filters, neural filters, and bilateral filters with and without various forms of classification in different enhancement applications including image de-blocking, noise reduction, and image interpolation. 
The rest of paper is organized as follows. We begin with an introduction about the nonlinear filters in Sect. 2. According to the way the nonlinear filters introduce nonlinearity, they can be classified into several categories. Four representative filters from these categories are reviewed and discussed in Sect. 3. In order to investigate the additional performance improvements with the content adaption, we apply these filters in the framework of content adaptive filtering and an evaluation of these nonlinear filters in different image processing applications are provided in Sect. 4. Finally, we draw our conclusion in Sect. 5.

\section{Categories of nonlinear filters}

The earliest and most widely used nonlinear filter probably is the median filter [8]. In the median filter, the median value in the filter window is the output of the filter. It shows good performance at removing impulsive noise and preserving edges [9]. In fact, the median filter uses the order statistics information, and the noisy values are regarded as outliers so that they can be removed. The further research about the median filter has led to a category of nonlinear filters that produce outputs based on the rank-ordered observations, such as order statistic (OS) filters [2,3]. Such filters based on only order statistics have some advantages over linear filters. They are robust in environments with impulsive interference, and they can track signal discontinuities without introducing smooth transitions, as linear filters do. However, the rank order information alone is not sufficient in many applications. To incorporate both the spatial order and rank order information, many generalizations of rank order filters have been proposed. Good examples among them are combination filters [10,23], permutation filters [11,12], and hybrid filters [13]. Different from the combination filters and the permutation filters that exhibit high complexity, the hybrid filter is relatively simple. The hybrid filter directly combines a linear filter and an OS filter. It exploits both the spatial and rank information in the image content and is proposed to realize the advantages of the OS filters in edge preservation and reduction of impulsive noise components while retaining the ability of the linear filter to suppress Gaussian noise.

With the introduction of the neural network to image processing, another type of nonlinear filters, the neural filter, has also been proposed [5,14]. The neural filter is essentially a multi-layer feed-forward neural network. The neural network takes the neighboring pixels from an image as the input and outputs the processed pixels. Rather than using the linear combination of the input pixel samples, a nonlinear transfer function at the hidden unit is applied to the weighted sum of the inputs. The flexibility of the neural network can be increased by using more hidden units or hidden layers. Because of its universal approximation property, the neu- ral network can provide a better function approximation by a supervised learning. With the more flexible nonlinear model, the neural filter has shown better performance than the linear filter [15].

The third category of nonlinear filters includes edgepreserving smoothing methods that utilize pixel similarity information. The early approaches, including the sigma filter [16] and the fuzzy filter [17], give the weights of input pixels according to their value differences from the central pixel value. More recently, the bilateral filter [4] has received considerable attention in areas of image processing and computer vision. Unlike the sigma filter and the fuzzy filter of which the coefficients are determined by the pixel value difference, the bilateral filter adjusts its coefficients to the spatial closeness and photometric similarity of the pixels. Due to this adaptivity, it has shown good performance at edge-preserving smoothing for image processing applications, such as noise reduction and digital coding artifact reduction [18]. For a linear filter, its coefficients can be adjusted to achieve desired effects by a supervised learning and the least mean square optimization. However, this is not trivial for the bilateral filter. In order to solve that problem, a new type of filter, the trained bilateral filter, is proposed [19]. The trained bilateral filter adopts a linear combination of spatially ordered and rank-ordered pixel samples, which has been proposed in a hybrid filter. Different from the hybrid filter where the similarity had been heavily quantized, the rank-ordered pixel samples in the proposed method are further transformed to reflect the photometric similarity of the pixels. Consequently, the trained bilateral filter possesses the essential characteristics of the original bilateral filter. On the other hand, the design of the proposed bilateral filter makes it feasible to optimize the filter coefficients. That is, the optimal coefficients for the combined pixel samples can be obtained by the least mean square optimization as for the linear filters.

\section{Representative nonlinear filters}

In this section, we choose four representative nonlinear filters from the mentioned categories in the previous section, the order statistics filter that only uses the rank order information, the hybrid filter that combines the rank order and spatial information, the trained bilateral filter that adopts both the spatial and similarity information, and the neural filter that introduces the nonlinear transfer function. The definitions and properties of these filters are then reviewed.

\subsection{Order statistic filter and hybrid filter}

Order statistics filters that are based on rank order information have been introduced to track signal discontinuities so that they can provide better edge preserving. However, using 
the rank order information alone fails to exploit the spatial context within the filter aperture. To incorporate both the spatial order information and the rank order information, the hybrid filter is proposed to combine a linear filter and an OS filter so that it can realize the advantages of both filters.

Let us start with the definition of a linear filter. Let $X=$ $\left(x_{1}, x_{2}, \ldots, x_{n}\right)^{T}$ be an observation containing $n$ samples arranged by the spatial or temporal order in which the samples are observed. $X_{r}$ is the sorted observation vector $X_{r}=$ $\left(x_{(1)}, x_{(2)}, \ldots, x_{(n)}\right)^{T}$ where $x_{(i)}$ is the $i$ th largest sample in $X$, so that $x_{(1)} \leq x_{(2)} \leq \cdots \leq x_{(n)}$. Let the observation vector $X$ be the input to the filter. For the linear filter, we have

$y=W^{T} X$

where $y$ is the output of the linear filter, and $W$ is an $N \times 1$ vector of coefficients for the linear filter. Consequently, the linear filter only takes consideration of the spatial position of the pixel samples.

Then, for an OS filter, we have

$y_{r}=W_{r}^{T} X_{r}$

where $y_{r}$ is the output of the OS filter, and $W_{r}$ is an $N \times 1$ vector of coefficients for the OS filter.

By concatenating $X$ and $X_{r}$, we can obtain an extended vector $X_{h}$ which contains spatial ordered and rank-ordered samples.

$X_{h}=\left(x_{1}, x_{2}, \ldots, x_{n}, x_{(1)}, x_{(2)}, \ldots, x_{(n)}\right)^{T}$

The hybrid filter is a linear combination of both spatial ordered and rank-ordered samples as shown in Eq. 4.

$y_{h}=W_{h}^{T} X_{h}$

where $y_{h}$ is the output of the hybrid filter, and $W_{h}$ is a $2 N \times 1$ vector of coefficients for the hybrid filter.

As one can see from Eq. 4, if the coefficients for the spatial ordered samples or the rank-ordered samples are constrained to be zero, the hybrid filter becomes equal to the OS filter or the linear filter, respectively.

The optimization of the hybrid filter can be accomplished in a similar fashion as for the linear filter. Suppose the output of the hybrid filter $y_{h}(t)=W_{h}^{T} X_{h}(t)$ is used to estimate the desired signal $d(t)$. The optimal filter coefficients are obtained when the mean square error between the output and desired signal is minimized. The mean square error MSE is:

$\mathrm{MSE}=E\left[\left(y_{h}(t)-d(t)\right)^{2}\right]=E\left[\left(W_{h}^{T} X_{h}(t)-d(t)\right)^{2}\right]$.
Taking the first derivative with respect to the weights and setting it to zero, we obtain [20]:

$W_{h}^{T}=E\left[X_{h} X_{h}^{T}\right]^{-1} E\left[X_{h} d\right]$

\subsection{Trained bilateral filter}

The trained bilateral filter is inspired by the bilateral filter and the hybrid filter. The bilateral filter is proposed as a generalization of other edge-preserving smoothing filters, such as the sigma filter [16] and the fuzzy filter [17]. It adapts its coefficients to the spatial closeness and photometric similarity of the pixels. Consequently, it shows very good performance at edge-preserving smoothing. The output $y_{b}$ of a bilateral filter is defined by [4]:

$y_{b}=\frac{\sum_{i=1}^{N} x_{i} \cdot c\left(x_{i}, x_{c}\right) \cdot s\left(x_{i}, x_{c}\right)}{\sum_{i=1}^{N} c\left(x_{i}, x_{c}\right) \cdot s\left(x_{i}, x_{c}\right)}$

$s\left(x_{i}, x_{c}\right)=\exp \left[-\left(x_{c}-x_{i}\right)^{2} / 2 \sigma_{s}^{2}\right]$

$c\left(x_{i}, x_{c}\right)=\exp \left[-d\left(x_{c}, x_{i}\right)^{2} / 2 \sigma_{c}^{2}\right]$

$i=1,2, \ldots, N$.

where $x_{c}$ is the spatially central pixel, and $d\left(x_{c}, x_{i}\right)$ is the Euclidean distance between the pixel position of $x_{i}$ and $x_{c}$. The Gaussian function has been typically used to relate coefficients to the geometric closeness and photometric similarity of the pixels, which seems somewhat arbitrary. Also, it is not obvious how to optimize the bilateral filter using a supervised learning like the hybrid filter.

As one can see, the hybrid filter incorporates both the rank order and spatial position information as the bilateral filter. However, the rank ordering in the hybrid filter only gives some indications of the pixel similarity, that is, the similarity has been heavily quantized. In order to incorporate the complete similarity information as the original bilateral filter does, we obtain the vector $X_{S}=\left(x_{[1]}, x_{[2]}, \ldots, x_{[N]}\right)^{T}$ by sorting the pixels in the filter aperture according to their pixel value distance to the spatially central pixel $x_{c}$. The ordering is defined by:

$\left|x_{[i+1]}-x_{c}\right| \geq\left|x_{[i]}-x_{c}\right|, \quad i=1,2, \ldots, N$.

Then, we transform the vector $X_{s}$ into $X_{s}^{\prime}=\left(x_{[1]}^{\prime}, x_{[2]}^{\prime}, \ldots\right.$, $\left.x_{[N]}^{\prime}\right)^{T}$. The transform is defined as:

$\begin{aligned} x_{[i]}^{\prime} & =\mu\left(x_{c}, x_{[i]}\right) \cdot x_{c}+\left(1-\mu\left(x_{c}, x_{[i]}\right)\right) \cdot x_{[i]}, \\ i & =1,2, \ldots, N .\end{aligned}$ 
where $\mu\left(x_{c}, x_{[i]}\right)$ is a membership function between $x_{[i]}$ and $x_{c}$. The membership function is defined as:

$\mu\left(x_{c}, x_{[i]}\right)=\operatorname{MIN}\left(\frac{\left|x_{[i]}-x_{c}\right|}{K}, 1\right)$.

where $K$ is a pre-set constant. Other membership functions such as a Gaussian function are also possible. The vector $X_{t b}$ is obtained by concatenating the vectors $X$ and $X_{s}^{\prime}$ :

$X_{t b}=\left(x_{1}, x_{2}, \ldots, x_{n}, x_{[1]}^{\prime}, x_{[2]}^{\prime}, \ldots, x_{[N]}^{\prime}\right)^{T}$.

Similar to the linear filter, we define the output of the proposed trained bilateral filter as:

$y_{t b}=W_{t b}^{T} X_{t b}$.

where $W_{t b}$ is a $2 N \times 1$ vector of weights.

The expected advantage of the trained bilateral filter is that the weights of the transformed samples that are similar to the center sample value are increased to better preserve edges and suppress the noise. On the other hand, the linear part obtains the spatial information that is useful for local image structure reconstruction. Essentially, the trained bilateral filter behaves as the original bilateral filter whose coefficients are continuously dependent on the spatial and intensity difference of pixels. Additionally, the coefficients of the trained bilateral filter can be optimized by a supervised learning in a similar fashion as the hybrid filter.

\subsection{Neural filter}

Different from other filters that use the rank order and similarity information, the neural filter introduces the nonlinearity by using a nonlinear transfer function. In the neural filter, a multi-layer feed-forward neural network is employed as a convolution kernel. The neural network takes the pixels in a filter window from the input image and outputs the processed pixel as the result of the neural network computation. A two-layer neural network with $N_{h}$ hidden units as shown in Fig. 1 is defined by:

$y_{n n}=f_{2}\left(L W f_{1}\left(I W X+b_{1}\right)+b_{2}\right)$.

where $I W$ is an $N_{h} \times N$ matrix of weights connecting the input layer to the hidden layer; $L W$ is a $1 \times N_{h}$ matrix of weights for the hidden layer; $b_{1}$ is an $N h \times 1$ matrix of bias for the hidden layer; $b_{1}$ is a bias for the output, and $f_{1}, f_{2}$ are transfer functions for the hidden and output layer, respectively. The transfer function can be an identity function or a sigmoid function. Functions such as the hyperbolic tangent that produce both positive and negative values are usually chosen for the hidden layer. Such functions tend to yield a faster training than functions that produce only positive values such as log-sigmoid, because of better numerical conditioning [21]. The identity function is often employed in the

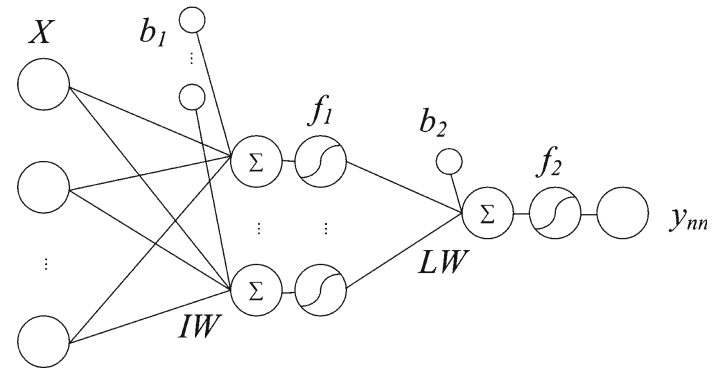

Fig. 1 The two-layer neural network model with several hidden units at the hidden layer

output layer because the characteristics of a neural network are improved significantly with an identity function when applied to function approximation issues in image processing [22]. When all the transfer functions are identity functions, the neural filter becomes a linear filter. The flexibility of the neural network can be increased by using more hidden units or hidden layers.

The neural network acquires various nonlinear functions by a supervised learning. The optimal coefficients for a neural network can be obtained through back-propagation [27]. During the training, the errors between outputs and targets are computed, and the derivatives of the errors are backpropagated to adjust the coefficients of the network iteratively and minimize the mean squared errors.

\section{Explicit content classification}

As one can see in the previous section, these nonlinear filters do not explicitly utilize the content classification. We expect that the content classification could bring additional performance improvement to these nonlinear filters. Therefore, we apply the nonlinear filters in the content adaptive filtering scheme as in [6]. As shown in Fig. 2, we replace the linear filtering part with a nonlinear filter. The local image structure within the filter aperture is first classified by the content classification. Then, an optimal linear filter is used to calculate the output pixel with filter coefficients fetched from the look-up-table (LUT). The coefficients of these nonlinear filters are optimized for every class.

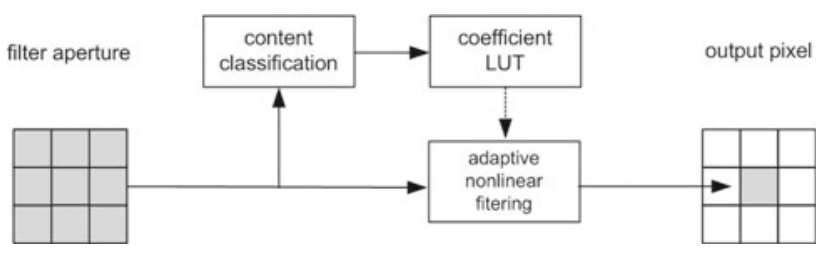

Fig. 2 The block diagram of using nonlinear filters in the proposed framework: the local image structure is classified using the content classification and the filter coefficients are obtained from the LUT 
In the following, an evaluation of the four mentioned nonlinear filters in different image processing applications, image de-blocking, noise reduction and image interpolation, is provided. In the evaluation, we compare these filters with the linear filter. And also different combinations of content classifications are investigated.

\subsection{Experiment setup}

\subsubsection{Training and test material}

The training material includes a variety of high quality natural images, including people, building, animals, and landscapes. All the filters are trained on the same training material. And the test images and the snapshots from the test sequences used in our experiments are shown in Fig. 3. Note that the test material is not included in the training material.

\subsubsection{Content classification}

For content classification, Adaptive Dynamic Range Coding (ADRC) [24] is a simple and efficient way to classify local image structures. The 1-bit ADRC code of every pixel is defined by:

$\operatorname{ADRC}\left(x_{i}\right)= \begin{cases}0, & \text { if } x_{i}<\frac{x_{\max }+x_{\min }}{2} \\ 1, & \text { otherwise }\end{cases}$

where $x_{i}$ is the value of pixels in the filter aperture, and $x_{\max }, x_{\min }$ are the maximum and minimum pixel value in the filter aperture. In the de-blocking application, ADRC classification is not enough to distinguish between coding artifacts and real image structures [25]. Therefore, one extra bit that includes the contrast information in the aperture, DR, can be

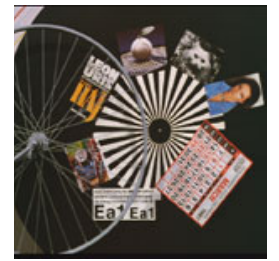

(A) Bicycle

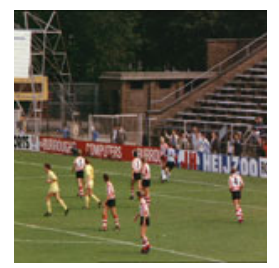

(D) Football

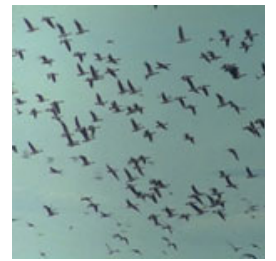

(B) Birds

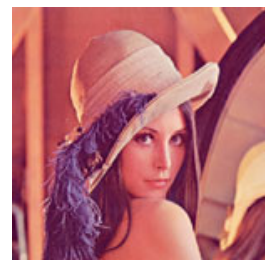

(E) Lena

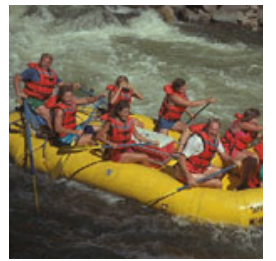

(C) Boat

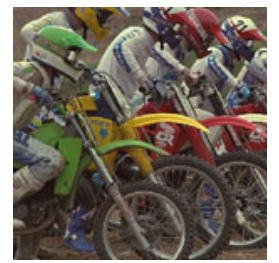

(F) Motor
Fig. 3 The testing material used for the evaluation added to the ADRC code. The extra bit DR is defined as:

$\mathrm{DR}= \begin{cases}0, & \text { if } x_{\max }-x_{\min }<T r \\ 1, & \text { otherwise }\end{cases}$

where $T r$ is the pre-set threshold value related to the coding quantization. The concatenation of $\operatorname{ADRC}\left(x_{i}\right)$ of all pixels in the filter aperture and the extra bit DR gives the class code, used to address the coefficient look-up table. The number of classes given by ADRC code can be reduced by bit inversion [24] to $2^{N-1}$. Together with the extra bit DR, the number of classes becomes $2^{N}$.

\subsubsection{Filter setting}

We use a diamond shape filter window shown in Fig. 4 to balance between the performance and the complexity.

For the neural filter setting, a two-layer feed-forward neural network is used. The transfer function used in the hidden layer is the hyperbolic tangent function, whereas the identity function is used at the output layer. The pixel value range in the neural filter is re-scaled from the range $[0,255]$ to $[-1$, 1], which corresponds to the output range of the hyperbolic tangent function. For a fair comparison, we use two hidden units in the hidden layer, which will result in a similar number of coefficients as the hybrid filter and the trained bilateral filter.

To give an impression of the filter complexity, Table 1 lists the numbers of the coefficients of different filters per class.

\subsubsection{Evaluation procedure}

For the evaluation, we degrade (compress, add noise, downscale) the original test sequence to generate the simulated input sequences. Then, different filters are applied to the sim-
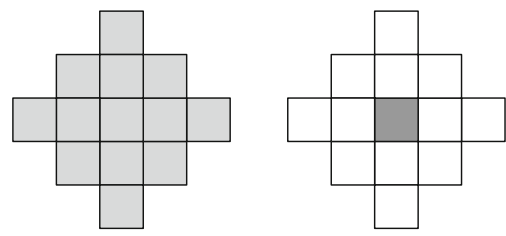

Fig. 4 The diamond shape filter window for de-blocking: the estimated output is in the center of the window

Table 1 Number of coefficients of different filters per class

\begin{tabular}{ll}
\hline Filter & Number of coefficients \\
\hline Linear & $\mathrm{N}$ \\
OS & $\mathrm{N}$ \\
Hybrid & $2 \mathrm{~N}$ \\
Trained bilateral & $2 \mathrm{~N}$ \\
Neural & $2 \mathrm{~N}+5$ \\
\hline
\end{tabular}


Table 2 MSE scores for de-blocking

\begin{tabular}{|c|c|c|c|c|c|c|c|c|c|c|c|c|}
\hline \multirow{2}{*}{$\begin{array}{l}\text { Sequence } \\
\text { Classification* }\end{array}$} & \multicolumn{4}{|l|}{ Linear } & \multicolumn{4}{|l|}{ OS } & \multicolumn{4}{|c|}{ Hybrid } \\
\hline & I & II & III & IV & I & II & III & IV & I & II & III & IV \\
\hline \multicolumn{13}{|l|}{ Mean square error } \\
\hline Birds & 11.6 & 11.6 & 11.3 & 11.8 & 17.2 & 17.3 & 14.6 & 13.6 & 11.5 & 11.5 & 11.0 & 10.9 \\
\hline Boat & 64.4 & 64.3 & 62.5 & 61.6 & 87.7 & 87.5 & 73.2 & 72.5 & 63.1 & 62.9 & 62.3 & 61.2 \\
\hline Lena & 31.9 & 31.9 & 30.2 & 30.1 & 39.4 & 39.3 & 34.0 & 33.3 & 31.1 & 31.1 & 30.2 & 29.6 \\
\hline Motor & 96.7 & 96.3 & 89.2 & 89.3 & 156.6 & 156.2 & 116.4 & 115.2 & 93.7 & 93.1 & 90.3 & 88.5 \\
\hline Average & 51.7 & 51.5 & 47.8 & 47.6 & 78.0 & 77.9 & 60.2 & 59.4 & 49.9 & 49.6 & 48.5 & 47.0 \\
\hline Sequence & \multicolumn{5}{|c|}{ Tr-bilateral } & \multicolumn{5}{|c|}{ Neural } & \multirow{2}{*}{\multicolumn{2}{|c|}{ Compressed }} \\
\hline Classification* & I & & II & III & IV & I & II & & III & IV & & \\
\hline \multicolumn{13}{|l|}{ Mean square error } \\
\hline Birds & 10.9 & & 10.9 & 10.5 & 10.5 & 11.2 & 11.2 & & 11.2 & 11.2 & 13.8 & \\
\hline Boat & 60.0 & & 59.9 & 59.5 & 59.5 & 63.4 & 62.8 & & 61.4 & 61.8 & 72.3 & \\
\hline Lena & 30.0 & & 29.9 & 29.0 & 28.8 & 31.9 & 31.0 & & 29.5 & 29.3 & 36.9 & \\
\hline Motor & 86.9 & & 86.8 & 84.2 & 84.2 & 95.4 & 94.3 & & 89.1 & 89.1 & 108. & \\
\hline Average & 46.6 & & 46.4 & 45.1 & 45.1 & 51.1 & 50.2 & & 47.4 & 47.3 & 59. & \\
\hline
\end{tabular}

* Classification: I, no classification, 1 class; II, DR, 2 classes; III, ADRC, 4096 classes; IV, ADRC+DR, 8192 classes

ulated input sequences. The MSE scores between the original test sequences and processed sequences will be used as the performance indicator.

\subsection{Image de-blocking}

In the experiment for image de-blocking, we evaluate the filter performance to remove JPEG compression coding artifacts. The test images and sequences have been compressed using JPEG compression at a quality factor of 20 (the quality factor of 100 is the best). The free baseline JPEG software from the Independent JPEG Group website ${ }^{1}$ is used for the JPEG encoding and decoding. As suggested in [25], we use the ADRC classification and the DR classification for the content classification. To show the contribution from the individual classifiers, we separately investigate the ADRC classification, the DR classification and their combination. For the DR classification, $T r=32$ is used.

In addition to the MSE score, we also use the BIM metric proposed by $\mathrm{Wu}[26]$ for the evaluation. The BIM metric measures the blockiness of compressed images or sequences. The $\mathrm{BIM}$ value $\mathrm{BIM}=1$ refers to no blockiness at all and the larger the BIM value is, the more blockiness in the content. A lower BIM value can be achieved by a strong smoothing filter. However, this will remove lots of details and increase the MSE score. Therefore, we use both the MSE and BIM scores for the evaluation.

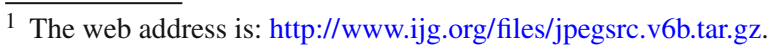

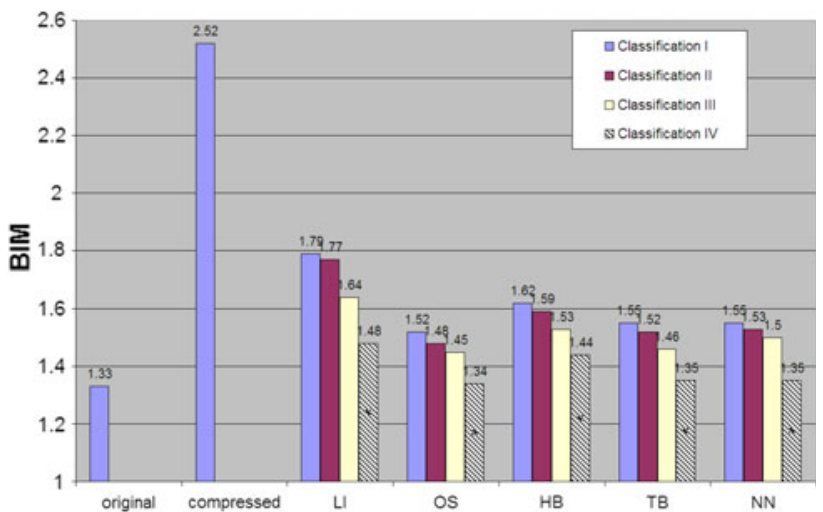

Fig. 5 The average BIM scores of the test sequences processed by these filters with different classifications: $L I$ Linear filter, $O S$ Order statistics filter, $H B$ Hybrid filter, $T B$ Trained bilateral filter, $N N$ Neural filter. Classification: I, no classification, 1 class; II DR, 2 classes; III ADRC, 4096 classes; IV ADRC+DR, 8192 classes

The MSE scores of all the filters with different classifiers are shown in Table 2. The average BIM scores of the test sequences processed by these filters are shown in Fig. 5. For the MSE score, all the nonlinear filters, except the OS filter, perform better than the linear filter. For the BIM score, all the nonlinear filters have better results than the linear filter, while the OS filter has the lowest BIM score. For both the MSE and BIM scores, all the filters can benefit from the ADRC classification and the DR classification. With the combination of the ADRC and DR classification, the best MSE and BIM scores are achieved. The OS filter has the highest MSE score because 


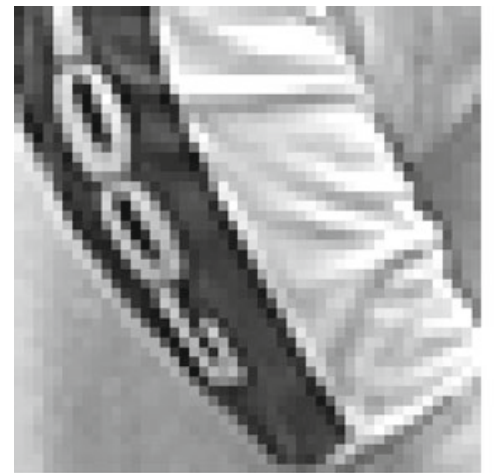

(A) Original

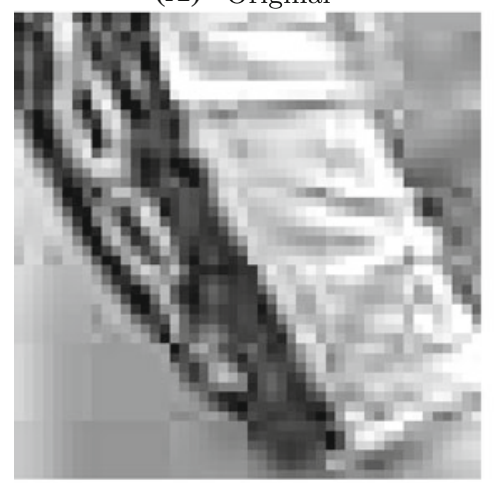

(B) Corrupted

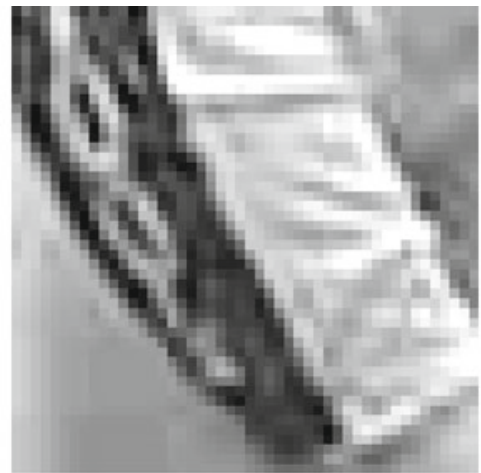

(C) LI

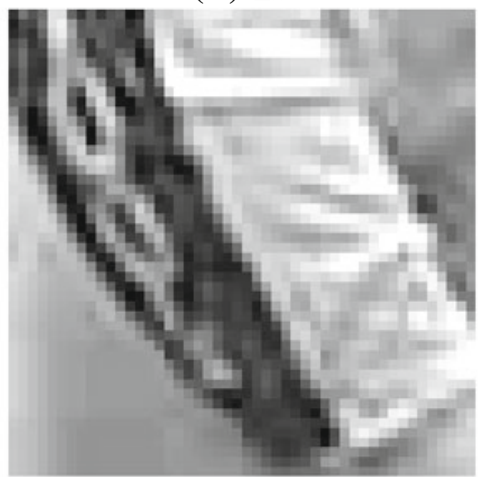

(D) LI DR

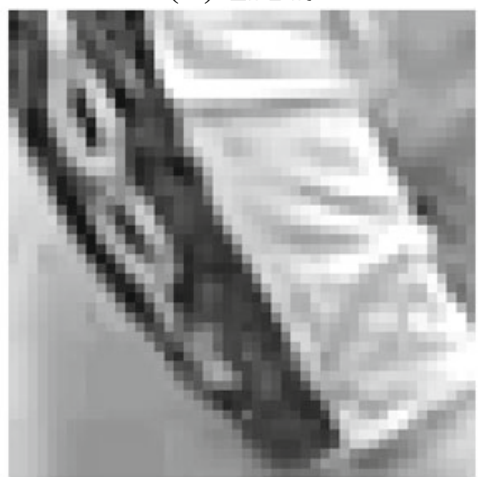

(E) LI ADRC

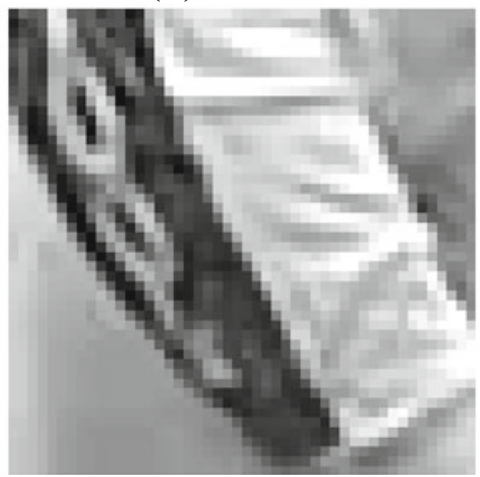

(F) LI ADRC+DR

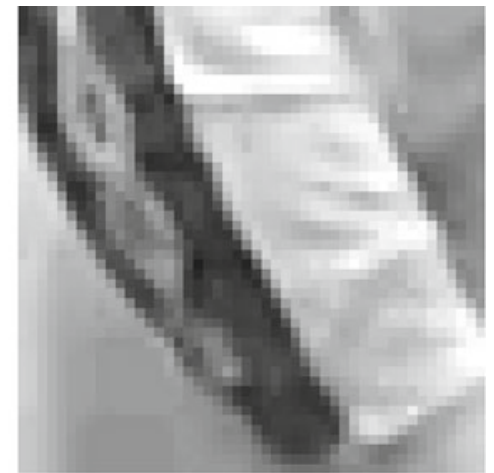

(G) OS

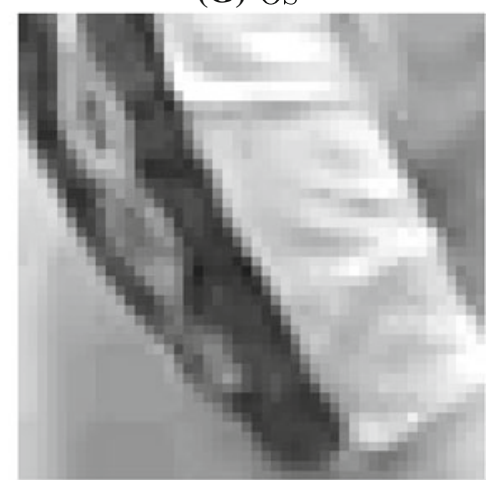

(H) OS DR

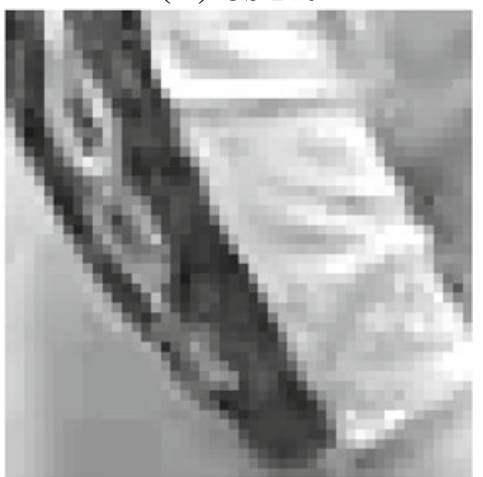

(I) OS ADRC

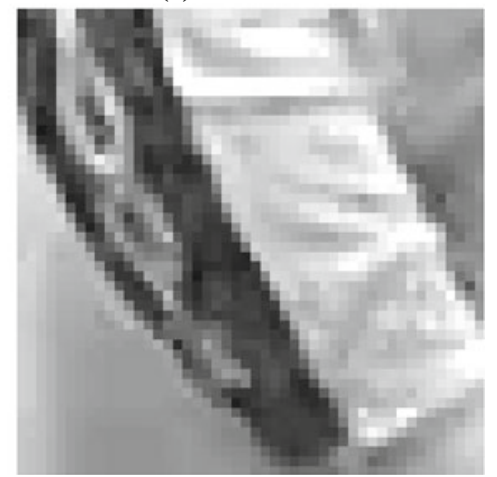

(J) OS ADRC+DR

Fig. 6 Image fragments from the image motor processed by different filters with different classifications: $L I$ Linear filter, $O S$ Order statistics filter 


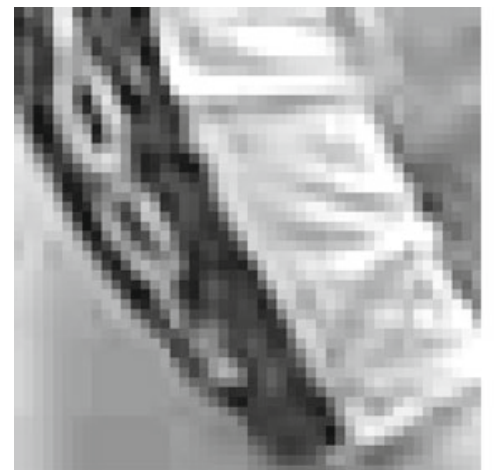

(K) $\mathrm{HB}$

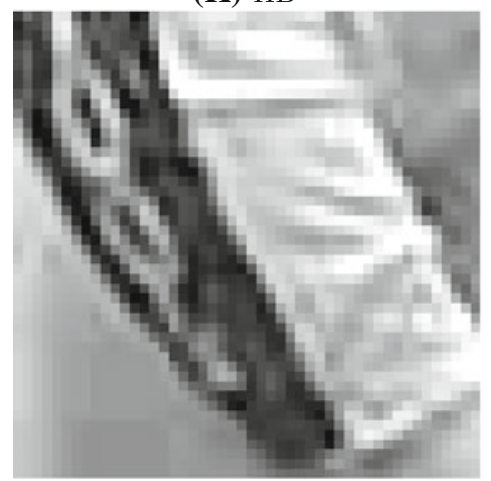

(L) HB DR

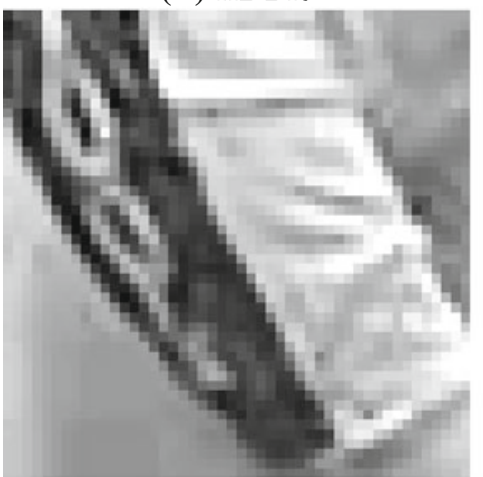

(M) HB ADRC

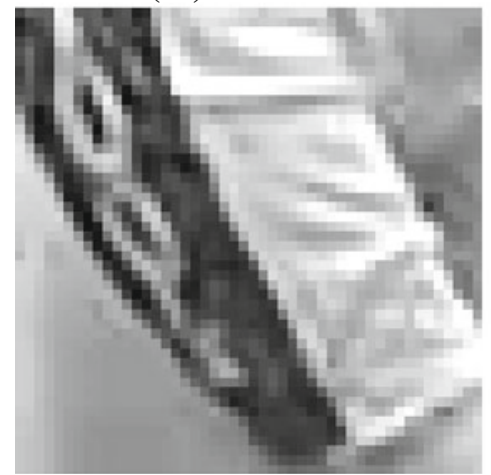

(N) HB ADRC+DR

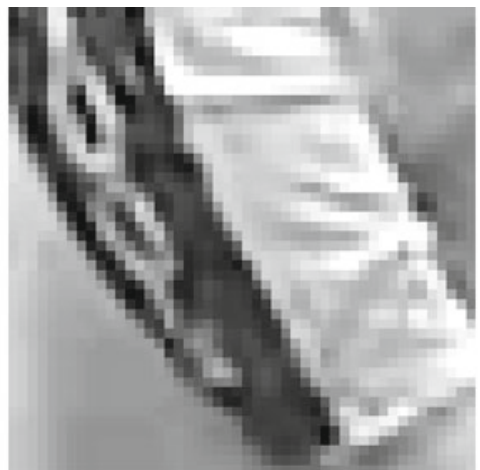

(O) $\mathrm{TB}$

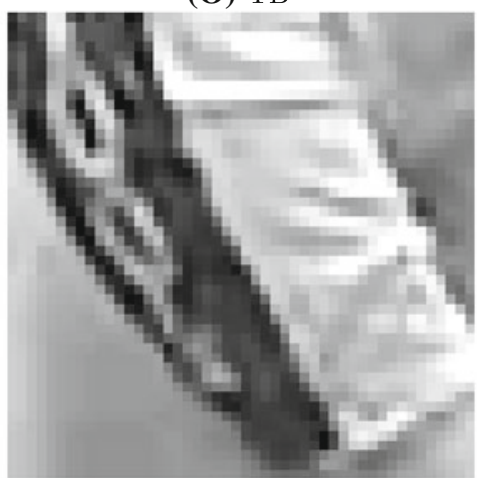

(P) TB DR

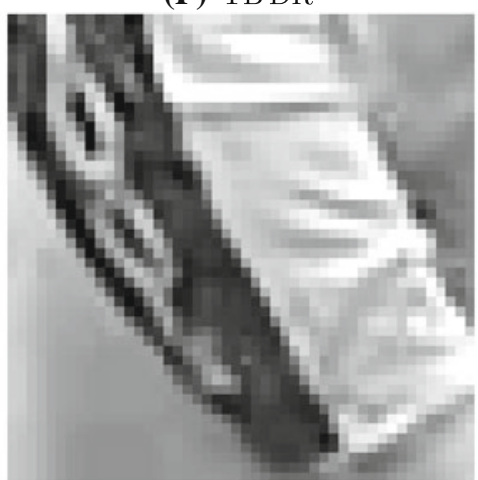

(Q) TB ADRC

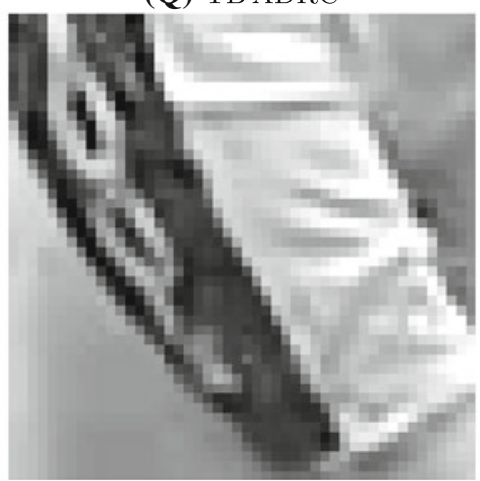

(R) $\mathrm{TB}$ ADRC+DR

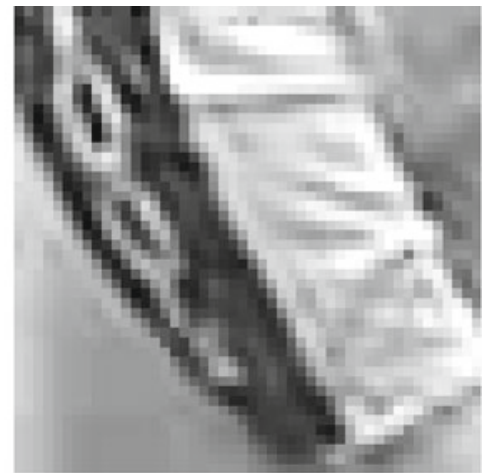

(S) $\mathrm{NN}$

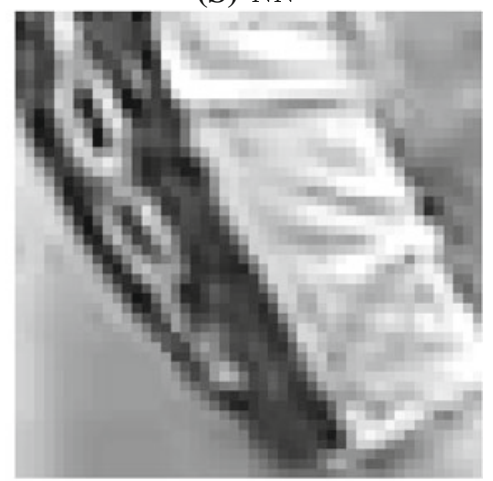

(T) NN DR

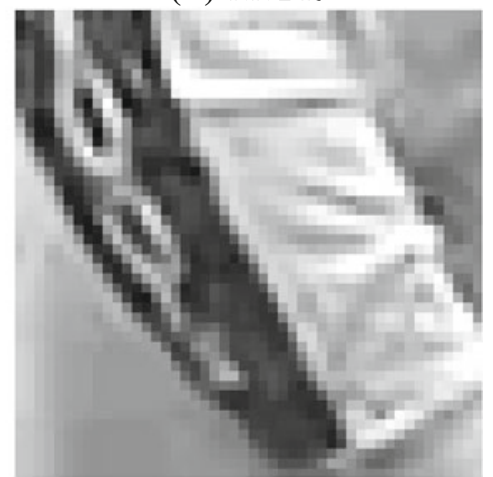

(U) NN ADRC

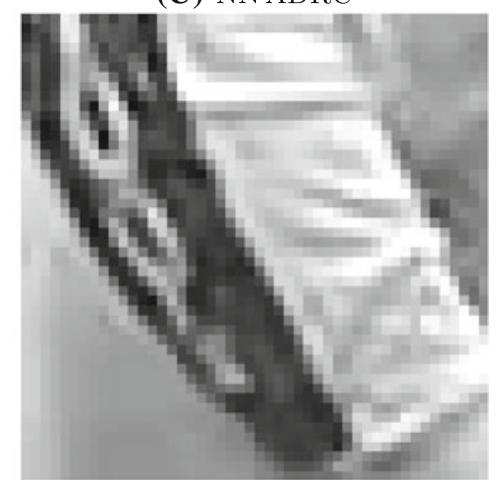

(V) NN ADRC+DR

Fig. 7 Image fragments from the image motor processed by different filters with different classifications: $H B$ Hybrid filter, $T B$ Trained bilateral filter, $N N$ Neural filter 
Table 3 MSE scores for noise reduction

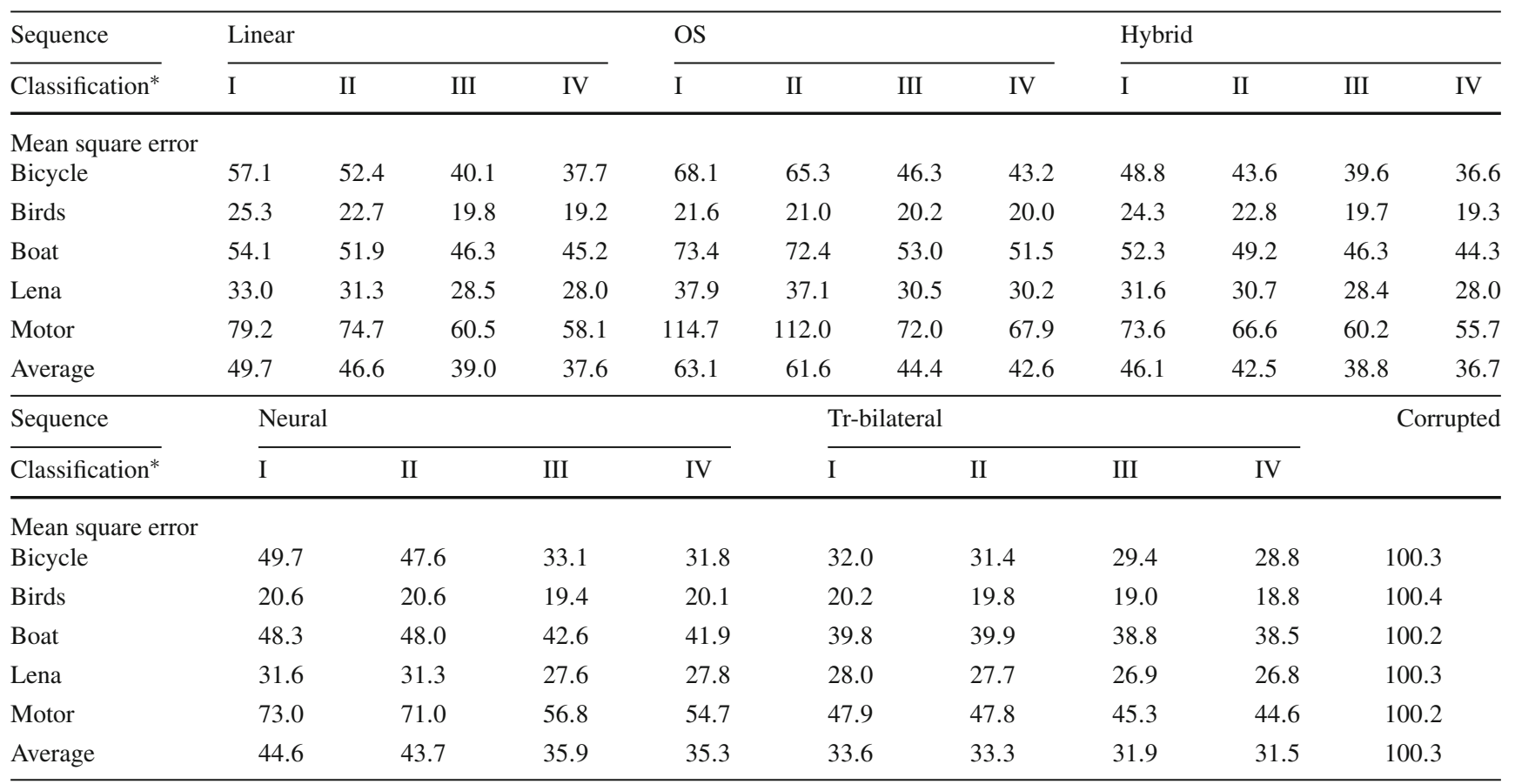

* Classification: I, no classification, 1 class; II, DR, 2 classes; III, ADRC, 4096 classes; IV, ADRC+DR, 8192 classes

it only uses the rank order information and fails to exploit the structure information. This can be shown in the MSE scores for the sequences such as Bicycle, Boat, and Motor which contain many image details. With the ADRC classification, the performance of the OS filter can be greatly improved, although it is still worse than the linear filter. The hybrid filter has shown better performance than the linear filter due to the added rank order information. With a similar complexity as the hybrid filter, the trained bilateral filter shows much better performance in the MSE score, even without the content classification. And it also achieves a relatively low BIM score. This suggests that the trained bilateral filter has a stronger signal adaptivity when the similarity information is incorporated so that the additional content classifications will not bring much improvement.

This is also reflected in the image fragments from Motor image processed by all the filters shown in Figs. 6 and 7. With the classification, the linear filter can suppress the coding artifact nicely, but the edges are also blurred comparing to the original. The OS filter can greatly reduce the coding artifacts, but it also destroys all the fine structural details. Although it shows better details preserving with the structure classification, the overall performance is not still as good as the linear filter. Comparing to the linear filter, both the hybrid filter and the neural filter can equivalently suppress the coding artifacts and demonstrate a better ability at preserving edges. As suggested in the MSE and BIM evaluation, the trained bilateral filter demonstrates the best edge-preserving ability and removes coding artifacts effectively. When comparing the results of using different classifications, we see that using the ADRC classification improves the performance at the fine details and using the ADRC+DR classification removes the blocking artifacts in the flat area better than using the ADRC classification alone.

\subsection{Noise reduction}

For noise reduction, we will evaluate these filters' abilities to remove Gaussian noise. The Gaussian noise usually manifests itself as irregular luminance patterns, which are different from real image structures. We expect that the ADRC classification could help distinguish the noise from the real image structures so that better noise reduction can be achieved. And also we hope that better noise reduction can be achieved in the low contrast area, in which case the DR classification is needed. Therefore, in the experiment, the content classifications, ADRC, DR, and ADRC+DR, are investigated. The Gaussian noise applied here has a mean of 0 and a standard deviation of 10 . The threshold used in the DR classification is optimized to $T r=40$.

Table 3 lists the MSE scores of all the methods in the applications of Gaussian noise reduction. Similar to the results of image de-blocking, all the filters can benefit from the ADRC classification and the DR classification. With the combination of the ADRC and DR classification, the best MSE scores 


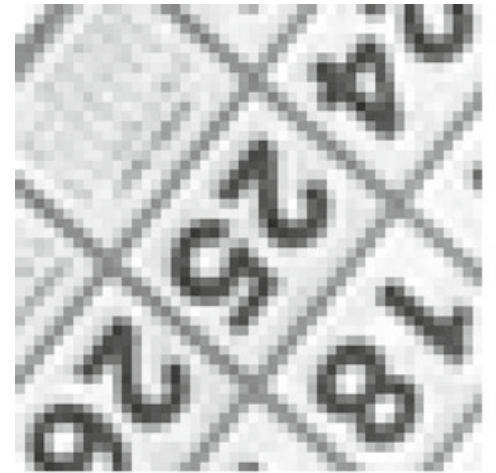

(A) Original

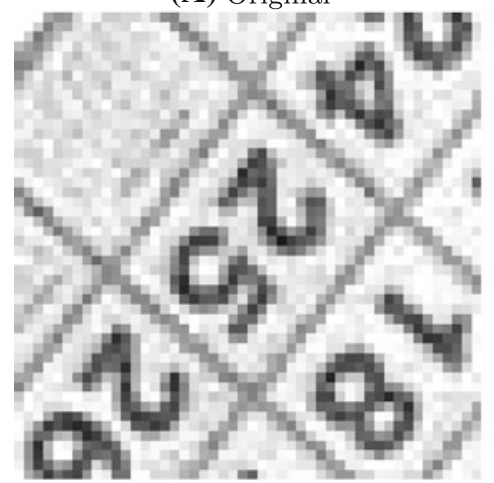

(B) Corrupted

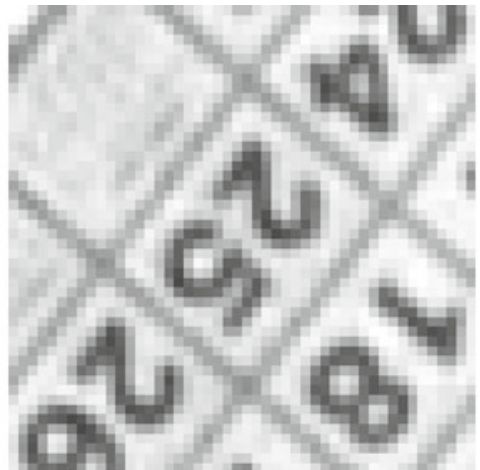

(C) LI

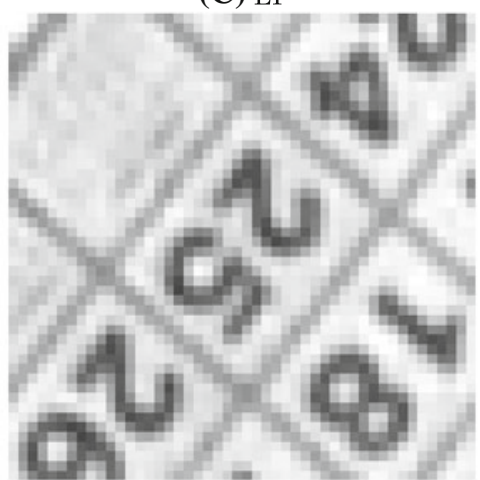

(D) LI DR

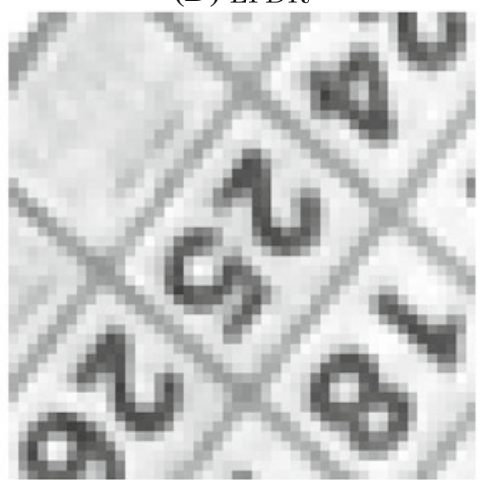

(E) LI ADRC

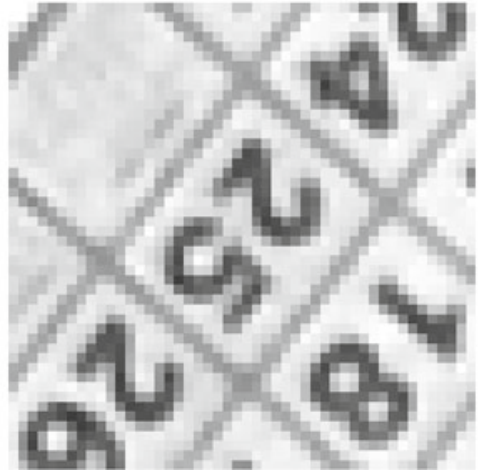

(F) LI ADRC+DR

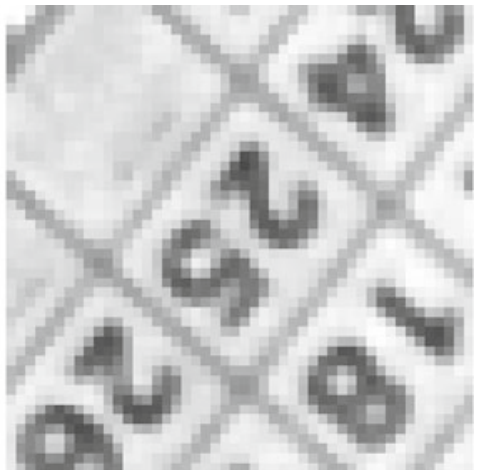

(G) $\mathrm{OS}$

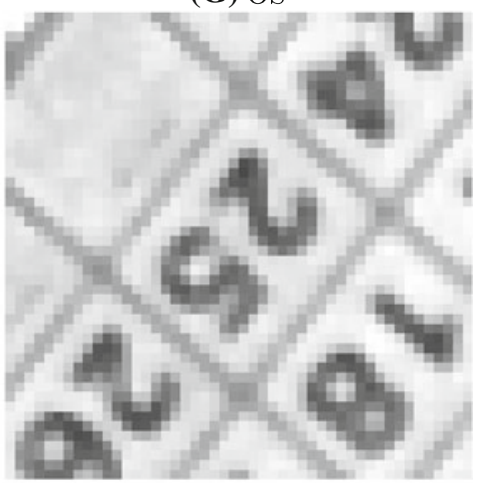

(H) OS DR

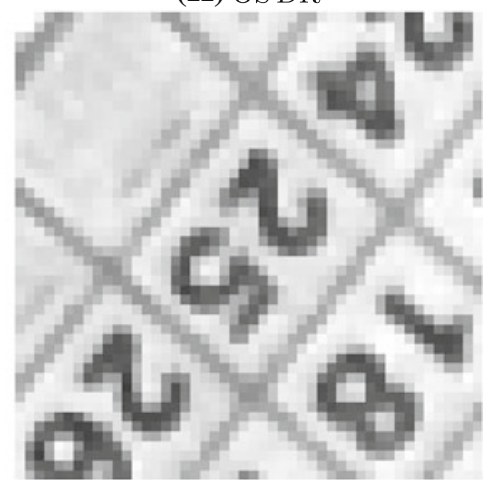

(I) OS ADRC

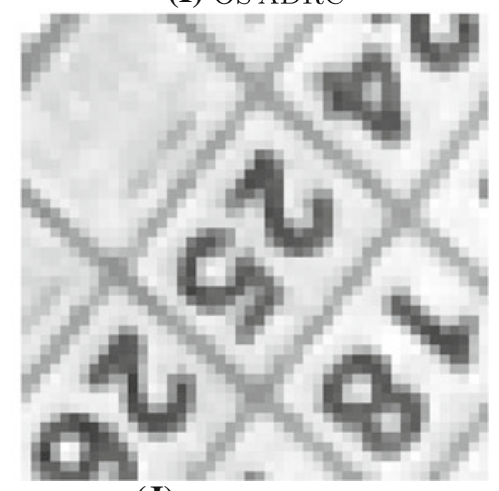

(J) OS ADRC+DR

Fig. 8 Image fragments from the sequence Bicycle processed by different filters with different classifications: $L I$ Linear filter, $O S$ Order statistics filter 


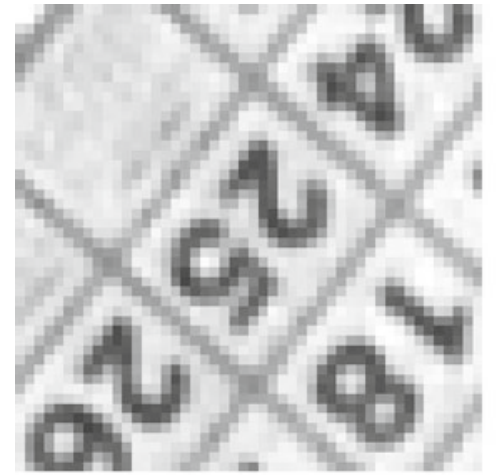

(K) $\mathrm{HB}$

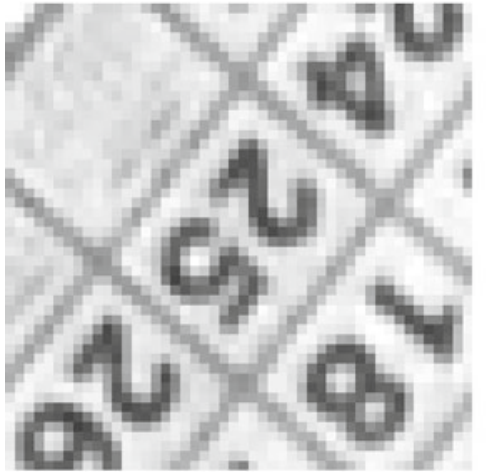

(L) HB DR

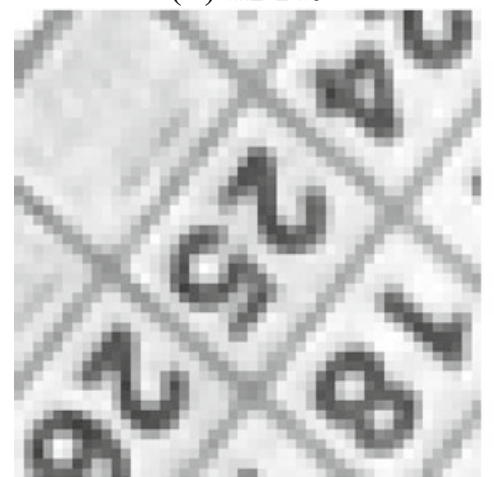

(M) HB ADRC

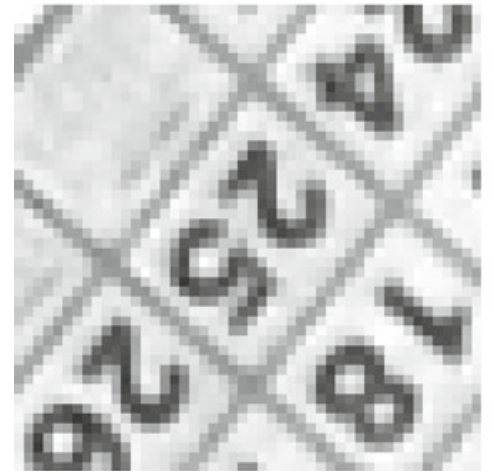

(N) $\mathrm{HB}$ ADRC+DR

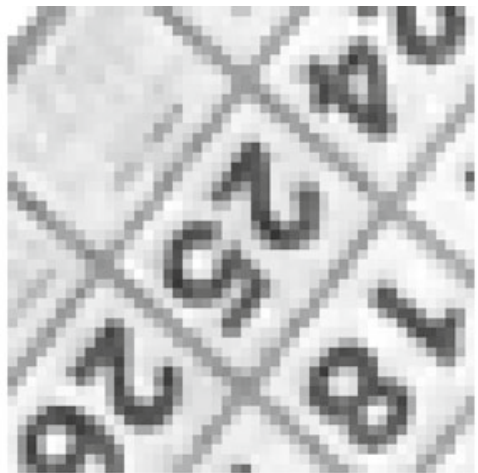

(O) $\mathrm{TB}$

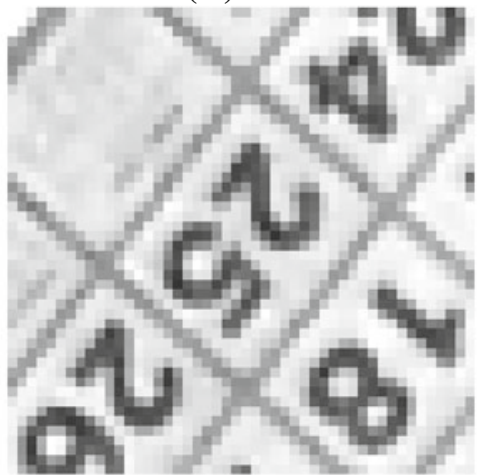

(P) TB DR

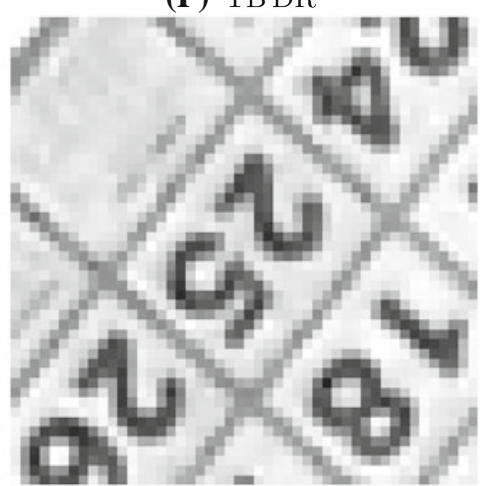

(Q) TB ADRC

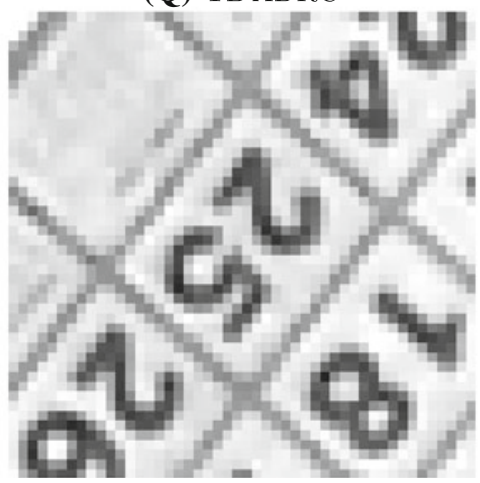

(R) $\mathrm{TB} A \mathrm{ARC}+\mathrm{DR}$

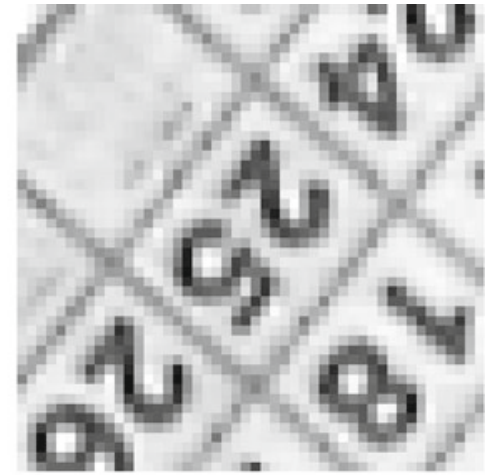

(S) $\mathrm{NN}$

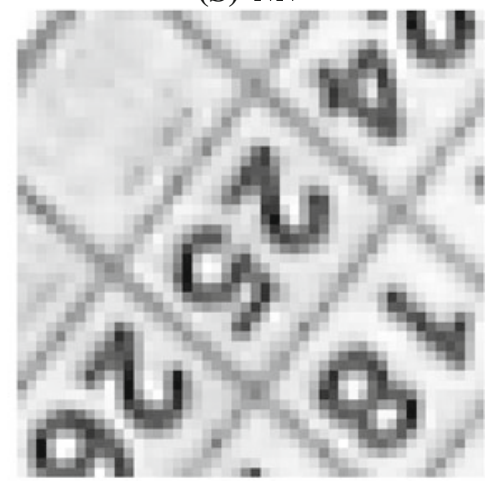

(T) NN DR

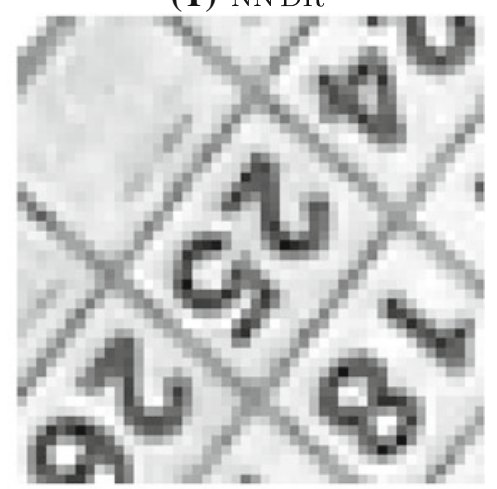

(U) NN ADRC

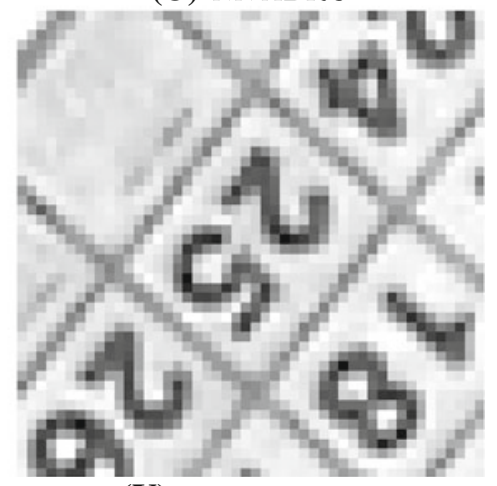

(V) NN ADRC+DR

Fig. 9 Image fragments from the sequence Bicycle processed by different filters with different classifications: $H B$ Hybrid filter, $T B$ Trained bilateral filter, $N N$ Neural filter 
Table 4 MSE scores for image interpolation

\begin{tabular}{|c|c|c|c|c|c|c|c|c|c|c|}
\hline \multirow{2}{*}{$\frac{\text { Sequence }}{\text { Classification* }}$} & \multicolumn{2}{|l|}{ Linear } & \multicolumn{2}{|l|}{ OS } & \multicolumn{2}{|c|}{ Hybrid } & \multicolumn{2}{|c|}{ Tr-bilateral } & \multicolumn{2}{|c|}{ Neural } \\
\hline & I & III & I & III & I & III & I & III & I & III \\
\hline $\begin{array}{l}\text { Mean square error } \\
\text { Bicycle }\end{array}$ & 68.2 & 45.4 & 291.8 & 78.0 & 68.4 & 44.5 & 68.3 & 43.2 & 62.3 & 42.1 \\
\hline Birds & 61.6 & 53.5 & 177.8 & 78.5 & 61.6 & 53.3 & 61.5 & 53.0 & 60.6 & 53.2 \\
\hline Boat & 64.7 & 56.6 & 268.3 & 85.7 & 64.9 & 57.9 & 64.6 & 55.8 & 63.3 & 54.5 \\
\hline Lena & 23.3 & 20.3 & 93.2 & 32.5 & 23.3 & 20.5 & 23.2 & 19.9 & 22.9 & 19.5 \\
\hline Motor & 108.7 & 85.8 & 331.1 & 134.6 & 108.8 & 84.5 & 108.7 & 86.8 & 103.1 & 85.4 \\
\hline Average & 65.3 & 52.3 & 232.4 & 81.9 & 65.4 & 52.1 & 65.3 & 51.7 & 62.4 & 50.9 \\
\hline
\end{tabular}

*Classification: I, no classification; 1 class, III, ADRC, 4096 classes

are achieved. Although the OS filter produces the worst MSE, with the content classification, it still has a quite close score to the linear filter. It suggests that the rank order information has some effect at removing the noise. This is also shown in the results of the hybrid filter. The MSE score of the hybrid filter is improved by combining the linear filter and the OS filter. The trained bilateral filter has a significant improvement over the hybrid filter, given the fact that they have a similar complexity. Without any content classification, the trained bilateral filter achieves a better MSE score than any other filter with the content classification.

To enable a qualitative comparison, some image fragments from the sequence Bicycle restored by all the filters are shown in Figs. 8 and 9. The OS filter shows a strong noise reduction, but it also removes the details. Although the edge-preserving performance of the OS filter can be further improved by the content classification, it is still not as good as the other nonlinear filters. The hybrid filter shows better performance at preserving edges than the linear filter. The trained bilateral filter further improves the edge preserving, producing the best contrast. The neural filter shows similar edge preserving, but it also produces some overshoots near the edges. When comparing the results of using different classifications, we see that using the DR classification improves the contrast a little and using the ADRC classification improves the performance at reconstructing the fine details. Furthermore, we see that the trained bilateral filter shows a great flexibility. It has good performance no matter whether the content classification is included. Without the content classification, it shows better performance than the linear filter with the content classification.

From the results of image de-blocking and noise reduction, we can conclude that similarity information is very useful for noise reduction applications. The rank information only gives some indication about the pixel similarity; therefore, the hybrid filter profits little from it. Although the neural filter is regarded as a flexible model which can approximate any smooth function, it still heavily depends on the content classification to get satisfactory results. From the results, we can see that a filter that is designed to inherently adapt to signal can achieve similar performance as a non-inherently adaptive filter that is based on the content classification. It also suggests that the performance of the neural filter can be further improved by inherent adaptations like the trained bilateral filter.

\subsection{Image interpolation}

In image interpolation, local structure classification has proven to bring significant improvement for the linear filtering [28]. We expect that the nonlinear filter based on the structure classification can further be improved. Because interpolation does not change with the local contrast, we use only ADRC for the content classification. We adopt the same evaluation process as Zhao [28].

In Table 4, the MSE scores on the test images and sequence in the application of image interpolation are provided. The table shows that the OS filter has the highest MSE score because they only use the rank order information and fail to exploit the content structure. The MSE scores for these filters with the ADRC classification have a significant reduction compared to those without on every test image and sequence, which suggests that the structure information is important for interpolation. Comparing the results from the linear filter, the hybrid filter and the trained bilateral filter, we see that the rank order information and the similarity information do not bring much improvement as they do not contribute to better interpolation. The neural filter demonstrates a somewhat more robust estimation and achieves the lowest MSE score.

For a qualitative comparison, some image fragments from the Bicycle sequence interpolated by these methods are shown in Fig. 10. Without the ADRC classification, none of these filters produces satisfactory results, especially the OS filter destroys the local structure heavily. With the ADRC classification, more image details have been reconstructed due to the local structure information. The results from the 


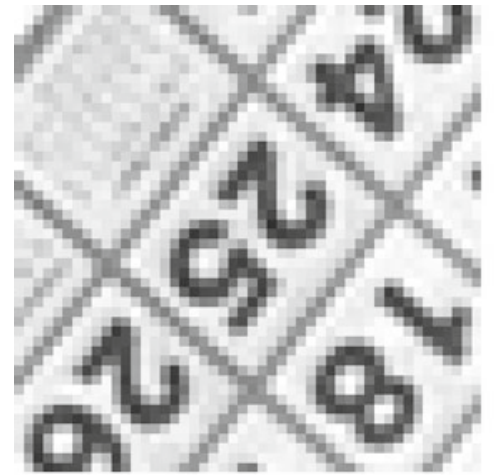

(A) Original

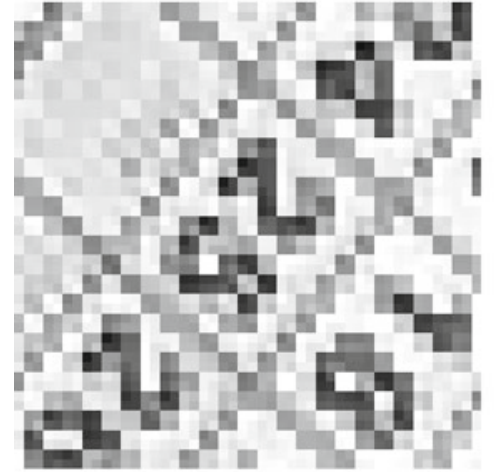

(B) Down-scaled

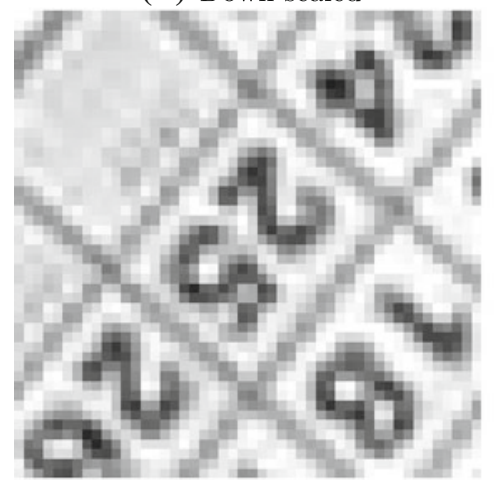

(G) $\mathrm{HB}$

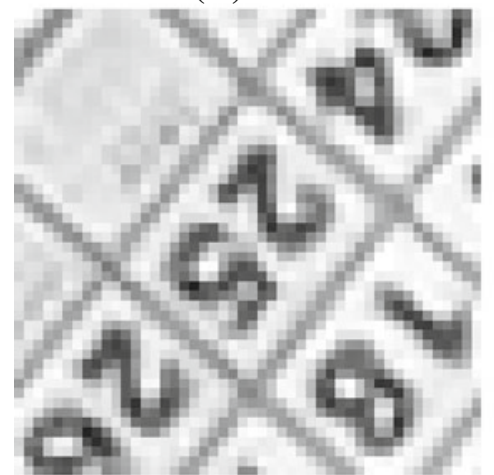

(H) HB ADRC

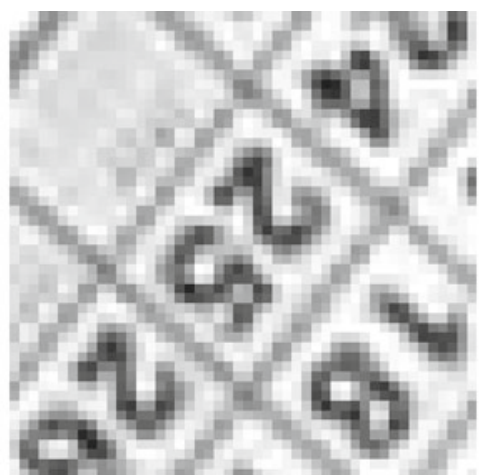

(C) LI

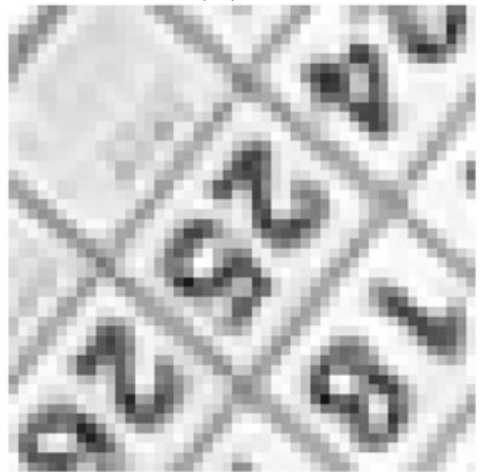

(D) LI ADRC

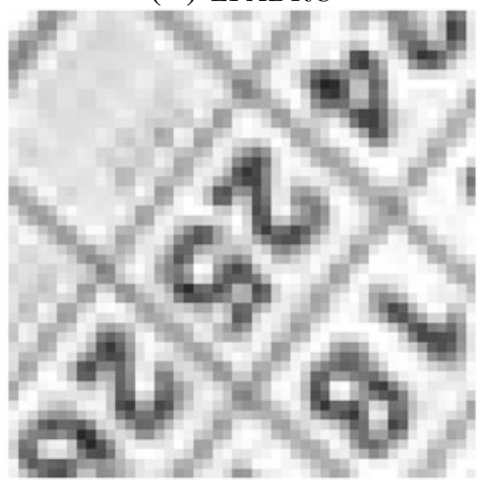

(I) $\mathrm{TB}$

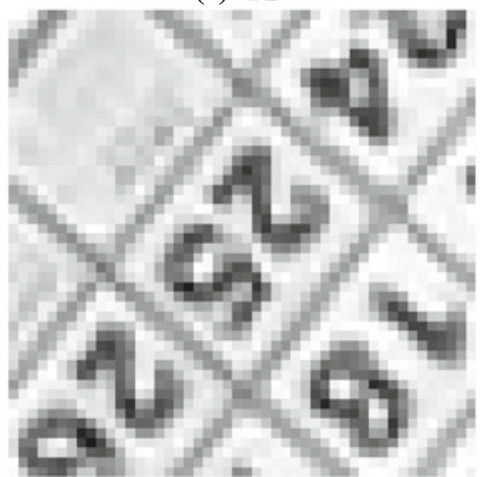

(J) TB ADRC

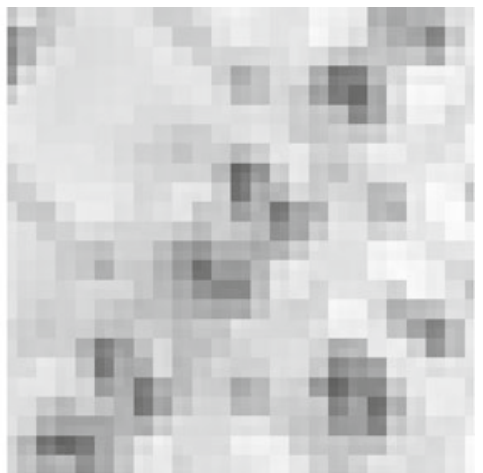

(E) OS

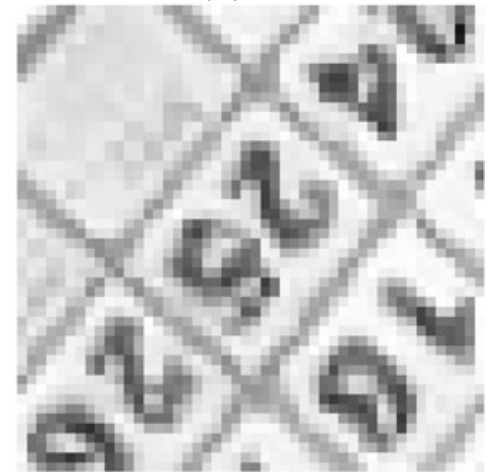

(F) OS ADRC

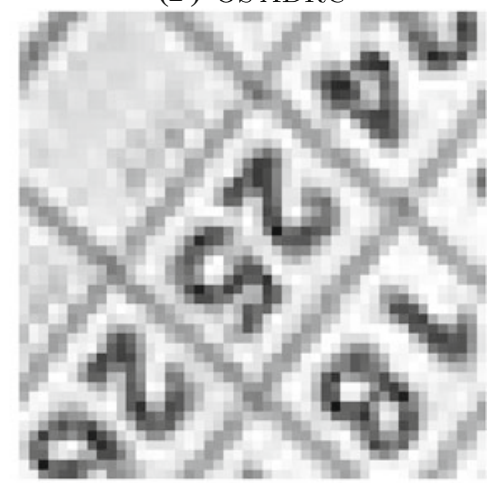

(K) NN

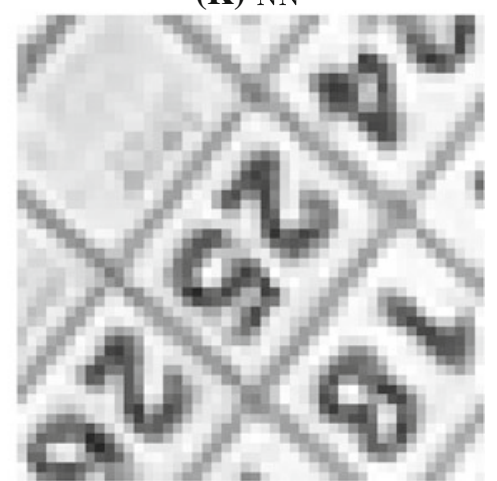

(L) NN ADRC

Fig. 10 Image fragments from the Bicycle sequence interpolated by different filters with different classifications: $L I$ Linear filter, $O S$ Order statistics filter, $H B$ Hybrid filter, $T B$ Trained bilateral filter, $N N$ Neural filter 
linear filter and the hybrid filter show that they generate some staircase effects at some lines, while those lines are reconstructed more smoothly by the trained bilateral filter and the neural filter. Comparing the results from the trained bilateral filter and the neural filter, we can also see that the neural filter reproduces thinner lines that are closer to the original.

\section{Conclusion}

In this paper, we have incorporated the explicit content classification to several types of nonlinear filters, the order statics filter, the hybrid filter which combines the linear and order statistics filter, the neural filter uses a nonlinear transfer function to introduce nonlinearity and the trained bilateral filter inspired by the bilateral filter and the hybrid filter. These nonlinear filters are applied in the framework of content adaptive filtering to see whether they can profit from the explicit content classification. A thorough evaluation of these nonlinear filters is given in image processing applications like image de-blocking, noise reduction, and image interpolation.

Since a linear filter has no inherent content adaptation, it benefits a lot from the explicit classification. The rank order filter has only a crude inherent adaptation using the ranking of input values. This crude adaptation allows rank order filters to heavily suppress outliers in the image, but also often destroys significant details. Our experiments show that this drawback can be greatly reduced with the explicit content classification. However, the result is still significantly worse than that of the linear filter with explicit classification in the applications we investigated. It should not come as a surprise therefore, that the hybrid filter, which basically combines the linear and rank order filter, can benefit from the classification but the result is only little better than that of the linear filter with classification.

The trained bilateral filter profits little from the explicit content classification as it already shows quite satisfactory implicit content adaptation. Our experiments suggest that for some applications (noise reduction), a nonlinear filter that inherently adapts well to the signal (bilateral filter) can perform similarly well as a simple linear filter with the content classification.

Although the neural filter apparently has a great flexibility, its performance can be greatly improved with additional content classification in the applications we tested. None of the filters we investigated has been specifically designed for image interpolation. Indeed, our experiments show that all filters can greatly benefit from explicit content classification.

Open Access This article is distributed under the terms of the Creative Commons Attribution Noncommercial License which permits any noncommercial use, distribution, and reproduction in any medium, provided the original author(s) and source are credited.

\section{References}

1. Yin, L., Yang, R., Gabbouj, M., Neuvo, Y.: Weighted median filters: a tutorial. IEEE Trans. Circuits Syst. 41, 157-192 (1996)

2. Bovik, A.C., Huang, T.S., Munson, D.C.: A generalization of median filtering using linear combinations of order statistics. IEEE Trans. Acoust. Speech Signal Process. ASSP-33, 13421349 (1983)

3. Lee, Y., Kassam, S.: Generalized median filtering and related nonlinear filtering techniques. IEEE Trans. Acoust. Speech Signal Process. 31, 672-683 (1985)

4. Tomasi, C., Manduchi, R.: Bilateral filtering for gray and color image. In: Proceedings of IEEE International Conference on Computer Vision, Bombay (1998, Sept)

5. Plaziac, N.: Image interpolation using neural networks. IEEE Trans. Image Process. 8(11), 381-390 (1999)

6. Kondo, T., Okumura, Y., Node, Y.: Picture conversion apparatus picture conversion method learning apparatus and learning method. US-Patent: US 6,323,905 B1, 27 Nov (2001)

7. Atkins, C.B., Bouman, C.A., Allebach, J.P.: Optimal image scaling using pixel classification. Int. Conf. Image Process. 3, 864$867(2001)$

8. Tukey, J.W.: Nonlinear methods for smoothing data. In: Conf. Rec. Eascon, pp. 673 (1974)

9. Brownrigg, D.R.K.: The weighted median filter. Commun. Assoc. Comput. Mach. 27(8), 807-818 (1984)

10. Gandhi, P.P., Kassam, S.A.: Design and performance of combination filters for signal restoration. IEEE Trans. Signal Process. 39, 1524-1540 (1991)

11. Barner, K.E., Arce, G.R.: Permutation filters: a class of nonlinear filters based on set permutations. IEEE Trans. Signal Process. 42, 782-798 (1994)

12. Hardie, R.C., Barner, K.E.: Extended permutation filters and their application to edge enhancement. IEEE Trans. Image Process. 5, 855-867 (1996)

13. Thompson, E.A., Hardie, R.C., Barner, K.E.: Hybrid order statistic filter and its application to image restoration. Appl. Opt. 40, 656$661(2001)$

14. Go, J., Sohn, K., Lee, C.: Interpolation using neural networks for digital still cameras. IEEE Trans. Consum. Electron. 46(3), 610$616(2000)$

15. Yin, L., Astola, J., Neuvo, Y.: A new class of nonlinear filters neural filters. IEEE Trans. Signal Process. 41(3), 1201-1222 (1993)

16. Lee, J.S.: Digital image smoothing and the sigma filter. Comput. Visi. Graph. Image Process. 24, 225-269 (1983)

17. Nie, Y., Barner, K.E.: Fuzzy transformation and its applications. IEEE International Conference on Image Processing, Barcelona (2003, Sept)

18. Wan, S., Mrak, M., Izquierdo, E.: Perceptually adaptive joint deringing deblocking filtering for scalable video coding. 2nd International Mobile Multimedia Communications Conference, Alghero (2006, Sept)

19. Hu, H., de Haan, G.: Trained bilateral filters and applications to coding artifacts reduction. In: Proceedings of IEEE International Conference on Image Processing, San Antonio, pp. 325-328 (2007, Sept)

20. Hu, H., de Haan, G.: Classification-based hybrid filters for image processing. In: Proceedings of SPIE, Visual Communications and Image Processing, San Jose (2006, Jan)

21. Fletcher, R.: Practical Methods of Optimization, 2nd edn. Wiley, pp. 100-107. ISBN 0471494631 (2000, Aug)

22. Suzuki, K., Horiba, I., Sugie, N.: Neural edge enhancer for supervised edge enhancement from noisy images. IEEE Trans. Pattern Anal. Mach. Intell. 25(12), 1582-1596 (2003) 
23. Palmieri, F., Boncelet, C.G. Jr.: Ll-filters-a new class of order statistic filters. IEEE Trans. Acoust. Speech Signal Process. 37, 691701 (1989)

24. Kondo, T., Fujimori, Y., Ghosal, S., Carrig, J.J.: Method and apparatus for adaptive filter tap selection according to a class. US-Patent: US 6,192,161 B1, 20 Feb (2001)

25. Hu, H., de Haan, G.: Simultaneous coding artifacts reduction and sharpness enhancement. In: Proceedings of IEEE International Conference on Consumer Electronics, Las Vegas (2007, Jan)
26. Wu, H.R.: A new distortion measure for video coding blocking artifacts. In: Proceedings of IEEE International Conference on Communication Technology, vol. 2, pp. 658-661 (1996)

27. Rojas, R.: Neural Networks-A Systematic Introduction. Springer, Berlin. ISBN 3540605053 (1996)

28. Zhao, M., Leitao, J.A., de Haan, G.: Towards an overview of spatial up-conversion techniques. In: Proceedings of ISCE'02, pp. E13E16 (2002, Sept) 